\title{
Modeling and Application of a New Nonlinear Fractional Financial Model
}

\author{
Yiding Yue, Lei He, and Guanchun Liu \\ Business School, Central South University, Changsha, Hunan 410083, China \\ Correspondence should be addressed to Lei He; helei19850716@csu.edu.cn
}

Received 7 May 2013; Accepted 23 October 2013

Academic Editor: Roberto Barrio

Copyright (C) 2013 Yiding Yue et al. This is an open access article distributed under the Creative Commons Attribution License, which permits unrestricted use, distribution, and reproduction in any medium, provided the original work is properly cited.

The paper proposes a new nonlinear dynamic econometric model with fractional derivative. The fractional derivative is defined in the Jumarie type. The corresponding discrete financial system is considered by removing the limit operation in Jumarie derivative's. We estimate the coefficients and parameters of the model by using the least squared principle. The new approach to financial system modeling is illustrated by an application to model the behavior of Japanese national financial system which consists of interest rate, investment, and inflation. The empirical results with different time step sizes of discretization are shown, and a comparison of the actual data against the data estimated by empirical model is illustrated. We find that our discrete financial model can describe the actual data that include interest rate, investment, and inflation accurately.

\section{Introduction}

The financial and economical systems exhibit ubiquitous complex dynamics evidenced by large-amplitude and periodic fluctuations, which have attracted much attention recently [1-4]. However, studying the dynamics of finance behavior and economics life is very challenging mainly because of the fact that it is very difficult to identify the inner relationship between different financial and economic variables quantitatively. In the recent decade, several nonlinear models have been proposed to study periodic or chaotic behaviors in financial and economic systems since it is believed that finance and economics always illustrate nonlinearity. In particular, the complex dynamics of economic cycles using the van der Pol model is studied in [57]. The advantage of employing forced van der Pol equation to depict economic variable is that the introduction of a forcing function enables us to model the complex interdependence between an individual national economy and the international economy in an era of globalization and the impact of climate variations such as the annual solar cycle on seasonable fluctuations of various markets. In $[8,9]$, the problem of the existence of a finite lag between the accrual and the payment of taxes in a framework, where this type of lag has never been considered by the well-known
IS-LM model, is proposed. The qualitative study of the system of functional differential equations shows that the finite lag may give rise to a wide variety of dynamic behaviors. These works, together with many recent papers, lead the studying of the nonlinear dynamics of financial and economic systems to be more attractive. Most of the studies about nonlinear dynamics of financial and economic systems concentrate on the periodic or chaotic behaviors in it. References [10,11] propose a simplified macrofinancial model which consists of interest rate, investment demand, and inflation based on the economic theory and analyzes the balance, stable periodic, chaotic motion, and so forth. In [12], the complex motion in nonlinear dynamical systems by reconsidering the Goodwin's nonlinear accelerator model with periodic investment outlays is studied, and it is believed that transient chaotic dynamics are prevalent in nonlinear economic model. In general, since the chaotic dynamics has adverse influences on predicting economy reasonably and effectively, more and more related papers pay attention to analyze and control the chaotic phenomenon of nonlinear dynamics in financial and economic systems. However, in these above works, only integer order differential equations are investigated. In recent years, modeling the real-world problems by using fractional differential equations becomes more and more popular. 
Fractional differential equation means that the order of derivative in differential equation can be noninteger and even complex number. As an excellent methodology of modeling, fractional calculus gained considerable development in the recent forty years. It has been applied to many scientific and engineering fields and verified to be a powerful tool in modeling most physical processes with memory effect, which cannot be described well by integer-order integral and differential equations. For a comprehensive review of theory and applications of fractional calculus, we refer to [13-17]. The advantage of fractional derivative is that it has nonlocal property. The value of current state depends on both recent values and historical values of objective function. This excellent property is suitable for modeling many financial variable series, mainly because of the fact that the financial and economic variable series always exhibit time-dependent memory effect, such as interest rate, stock price, exchange amount of future, and so on [18-21]. There are only a few papers on the study of financial and economic behaviors by using fractional derivative models. In [22], a fractional order financial model based on the fractional Chen system is proposed. It involves the macroeconomic variables such as investment, interest, and price index and exhibits the interesting dynamics behavior of them. In [23, 24], the chaos dynamics and chaos control have been studied using sliding mode method and feedback control method, respectively. In [25], a delayed fractional-order financial system is proposed and the complex dynamical behaviors of this system are discussed by numerical simulations. A great variety of interesting dynamical behaviors of such a system including single-periodic, multiple-periodic, and chaotic motions are shown. The effect of time delay and fractional order on the chaotic behavior are investigated; it is verified that a proper time delay can enhance or suppress the emergence of chaos.

In most of the recent researches, the dynamics of fractional-order financial and economic systems has been investigated via several mathematical methods (see [22, 25] and references therein). However, the physical and natural interpretations of those dynamical phenomena obtained in numerical simulations are still not clear. We have known that the occurrence of chaos in fractional-order financial system depends on both parameters and fractional order, but there is no particular study which concentrates on how to select the proper fractional order and parameters for a concrete group of economic data. For instance, although we have several methods to control the chaos in the fractionalorder financial systems, we have no idea what the economic interpretation of these procedures of controlling chaos is. In practice, the financial and economic data are determined with inner randomness of the real-world issues. Consequently, there should exist a group of corresponding parameters and fractional order, which can be used for depicting the particular financial and economic variables. In this paper, we propose a new nonlinear dynamical model of financial and economic systems by econometric techniques. First of all, we discretize the fractional derivative defined by Jumarie's definition and then derive the estimation of parameters in the model based on the least squared principle. Finally, we will take the macroeconomic data of Japan, for an example, to study the reasonability of the econometric model empirically.

To close this introduction, we would like to make several remarks. First, the study of financial and economic models which are simplified gained much interest in recent years both from integer and fractional calculus. However, most of them deal with the mathematical properties of the models and reveal the dynamic behaviors in numerical simulations, which lead those results, although very beautiful in mathematical point of view, to be very difficult to apply in real finance or economic life. Second, we are not going to employ the existing financial models but construct a new discrete financial model for depicting the real financial and economic data from a particular country, that is, Japan. The suitable parameters and fractional orders are determined by considering the macroeconomic data of it. Finally, based on the novel discrete financial model, we can analyze fitting effectiveness of the empirical model. To the authors' knowledge, this is the first time that the realistic financial and economic data of particular country are modeled by using continuous or discrete fractional-order nonlinear dynamic financial model. The remainder of this paper is organized as follows. In Section 2, the mathematical preliminaries are introduced. In Section 3, the nonlinear dynamic econometric model of financial system with fraction-order derivative is proposed, and the parameters of the nonlinear fractional-order financial model are estimated by least squares principal. In Section 4, the empirical study of macroeconomic data of Japan is shown. Finally, the conclusions are given in Section 5.

\section{Mathematical Preliminaries}

In this section, we introduce some preliminaries of fractional derivative. More properties of fractional derivatives could be found in the books and recent papers; for example, see [14, 26, 27].

Let $f(x): \mathbb{R} \mapsto \mathbb{R}$ denote a continuous function, and let $h>0$ denote a constant discretization span. The fractional difference of order $\alpha(\alpha \in \mathbb{R}, 0<\alpha \leq 1)$ of $f(x)$ is defined as follows (see [27]):

$$
\Delta^{\alpha} f(x):=\sum_{k=0}^{+\infty}(-1)^{k}\left(\begin{array}{l}
\alpha \\
k
\end{array}\right) f(x+(\alpha-k) h),
$$

and then its fractional derivative of order $\alpha$ is defined by

$$
D_{t}^{\alpha} f(t)=\lim _{h \rightarrow 0} \frac{\Delta^{\alpha} f(t)}{h^{\alpha}} .
$$

In [27], the Jumarie's modified fractional derivative of order $\alpha$ for a continuously differentiable function $u$ : $[0,+\infty) \rightarrow \mathbb{R}$ is defined as

$$
{ }^{*} D_{t}^{\alpha} f(t)=\Gamma(1+\alpha-m) \lim _{h \rightarrow 0} \frac{\Delta f^{(m)}(t)}{h^{\alpha-m}},
$$

where $m<\alpha<m+1, m=[\alpha],[\alpha]$ denotes the integer part of the real number $\alpha$. Furthermore, if $0<\alpha<1$, then

$$
{ }^{*} D_{t}^{\alpha} f(t)=\Gamma(1+\alpha) \lim _{h \rightarrow 0} \frac{\Delta f(t)}{h^{\alpha}} .
$$


There are several definitions of fractional derivative in [14], but in this paper we only use the Jumarie's fractional derivative. The advantage of applying Jumarie's fractional derivative is that we can select a small step size $h$ and remove the limit operation in (4); then, the discrete form of fractional derivative can be represented by the classical difference of function, multiplied by some coefficients.

For simplicity in writing, we remove the star "*” in Jumarie's fractional derivative. In what follows, we construct our continuous and discrete financial model by using Jumarie's fractional derivative.

\section{Model Description and Estimation Methodology}

There exist several financial models reported in recent years. For instance, the study of investment, interest rate, and price index by using a chaotic fractional Chen system is discussed in $[22]$ as

$$
\begin{aligned}
& D_{t}^{q_{1}} x_{t}=z_{t}+\left(y_{t}-p\right) x_{t}, \\
& D_{t}^{q_{2}} y_{t}=1-b y_{t}-x_{t}^{2}, \\
& D_{t}^{q_{3}} z_{t}=-x_{t}-c z_{t},
\end{aligned}
$$

where $x, y$, and $z$ represent the interest rate, investment, and inflation respectively. The subscript $t$ indicates that the variable depends on $t$. Parameters $p, b$, and $c$ are nonnegative coefficients with economic interpretation. $q_{i} \in(0,1](i=$ $1,2,3)$ represent the fractional order of the derivatives. If $q_{1}=q_{2}=q_{3}=1$, (5) reduces to the integer-order Chen system.

Instead of considering the same expressions in fractional chaotic Chen system, we assume a more general form of the our financial model as

$$
\begin{aligned}
D_{t}^{q_{1}} x_{t}= & c_{1}+a_{11} x_{t}+a_{12} y_{t}+a_{13} z_{t}+a_{14} x_{t} y_{t} \\
& +a_{15} y_{t} z_{t}+a_{16} z_{t} x_{t}+a_{17} x_{t}^{2}+a_{18} y_{t}^{2} \\
& +a_{19} z_{t}^{2}+u_{1 t}, \\
D_{t}^{q_{2}} y_{t}= & c_{2}+a_{21} x_{t}+a_{22} y_{t}+a_{23} z_{t}+a_{24} x_{t} y_{t} \\
& +a_{25} y_{t} z_{t}+a_{26} z_{t} x_{t}+a_{27} x_{t}^{2}+a_{28} y_{t}^{2} \\
& +a_{29} z_{t}^{2}+u_{2 t}, \\
D_{t}^{q_{3}} z_{t}= & c_{3}+a_{31} x_{t}+a_{32} y_{t}+a_{33} z_{t}+a_{34} x_{t} y_{t} \\
& +a_{35} y_{t} z_{t}+a_{36} z_{t} x_{t}+a_{37} x_{t}^{2}+a_{38} y_{t}^{2} \\
& +a_{39} z_{t}^{2}+u_{3 t},
\end{aligned}
$$

where $u_{i t}(i=1,2,3)$ are the random errors which are assumed to the white noise generally. $x_{t}, y_{t}$, and $z_{t}$ indicate that the variables $x, y$, and $z$ depend on time $t$.

$$
\begin{aligned}
& \text { Let } \\
& \begin{aligned}
f\left(x_{t}, y_{t}, z_{t}, A_{1}\right)= & c_{1}+a_{11} x_{t}+a_{12} y_{t}+a_{13} z_{t}+a_{14} x_{t} y_{t} \\
& +a_{15} y_{t} z_{t}+a_{16} z_{t} x_{t}+a_{17} x_{t}^{2} \\
& +a_{18} y_{t}^{2}+a_{19} z_{t}^{2}, \\
g\left(x_{t}, y_{t}, z_{t}, A_{2}\right)= & c_{2}+a_{21} x_{t}+a_{22} y_{t}+a_{23} z_{t}+a_{24} x_{t} y_{t} \\
& +a_{25} y_{t} z_{t}+a_{26} z_{t} x_{t}+a_{27} x_{t}^{2} \\
& +a_{28} y_{t}^{2}+a_{29} z_{t}^{2}, \\
h\left(x_{t}, y_{t}, z_{t}, A_{3}\right)= & c_{3}+a_{31} x_{t}+a_{32} y_{t}+a_{33} z_{t}+a_{34} x_{t} y_{t} \\
& +a_{35} y_{t} z_{t}+a_{36} z_{t} x_{t}+a_{37} x_{t}^{2} \\
& +a_{38} y_{t}^{2}+a_{39} z_{t}^{2}, \\
A_{i}= & \left(c_{i}, a_{i 1}, a_{i 2}, \ldots, a_{i 9}\right), \quad i=1,2,3 ;
\end{aligned}
\end{aligned}
$$

then, the model can be rewritten as

$$
\begin{aligned}
& D_{t}^{q_{1}} x_{t}=f\left(x_{t}, y_{t}, z_{t}, A_{1}\right)+u_{1 t}, \\
& D_{t}^{q_{2}} y_{t}=g\left(x_{t}, y_{t}, z_{t}, A_{2}\right)+u_{2 t}, \\
& D_{t}^{q_{3}} z_{t}=h\left(x_{t}, y_{t}, z_{t}, A_{3}\right)+u_{3 t} .
\end{aligned}
$$

According to (4), when $0<\alpha<1$, the model (8) can be discretized as

$$
\begin{aligned}
& \frac{x\left(t_{n+1}\right)-x\left(t_{n}\right)}{\left(t_{n+1}-t_{n}\right)^{q_{1}}} \Gamma\left(1+q_{1}\right)=f\left(x_{t_{n}}, y_{t_{n}}, z_{t_{n}}, A_{1}\right)+u_{1 t}, \\
& \frac{y\left(t_{n+1}\right)-y\left(t_{n}\right)}{\left(t_{n+1}-t_{n}\right)^{q_{2}}} \Gamma\left(1+q_{2}\right)=g\left(x_{t_{n}}, y_{t_{n}}, z_{t_{n}}, A_{2}\right)+u_{2 t}, \\
& \frac{z\left(t_{n+1}\right)-z\left(t_{n}\right)}{\left(t_{n+1}-t_{n}\right)^{q_{3}}} \Gamma\left(1+q_{3}\right)=h\left(x_{t_{n}}, y_{t_{n}}, z_{t_{n}}, A_{3}\right)+u_{3 t} .
\end{aligned}
$$

We estimate (9) based on empirical data to determine the relationship of these variables. From the form of the model (9), it is easy to find that there do not exist common parameters in three equations of it. Therefore, the above three multivariate regression equations can be estimated separately. To state the technical procedures, we take the first equation as an example. The estimation for the parameters in the other two equations is similar.

Consider $q_{1}=1$; then

$$
\frac{x\left(t_{n+1}\right)-x\left(t_{n}\right)}{\left(t_{n+1}-t_{n}\right)}=f\left(x_{t_{n}}, y_{t_{n}}, z_{t_{n}}, A_{0}\right)+u_{1 t} .
$$

Let $Y_{i}=x\left(\left(t_{i+1}\right)-x\left(t_{i}\right)\right) /\left(t_{i+1}-t_{i}\right), i=1,2, \ldots, N-1$; then define the least squares (LS) function as

$$
\operatorname{SSR}\left(A_{0}\right)=\sum_{i=1}^{N-1}\left(Y_{i}-f\left(x\left(t_{i}\right)\right), y\left(t_{i}\right), z\left(t_{i}\right), A_{0}\right)^{2} .
$$


The LS estimator of the regression parameter $\widehat{A}_{0}$ is obtained by minimizing the $\operatorname{SSR}\left(A_{0}\right)$ with respect to $A_{0}$ :

$$
\widehat{A}_{0}=\arg \min \sum_{i=1}^{N-1}\left(Y_{i}-f\left(x\left(t_{i}\right)\right), y\left(t_{i}\right), z\left(t_{i}\right), A_{0}\right)^{2} .
$$

For simplicity, we denote $X_{1 i}=x\left(t_{i}\right), X_{2 i}=y\left(t_{i}\right), X_{3 i}=$ $z\left(t_{i}\right), X_{4 i}=x\left(t_{i}\right) y\left(t_{i}\right), X_{5 i}=y\left(t_{i}\right) z\left(t_{i}\right), X_{6 i}=z\left(t_{i}\right) x\left(t_{i}\right), X_{7 i}=$ $x\left(t_{i}\right)^{2}, X_{8 i}=y\left(t_{i}\right)^{2}, X_{9 i}=z\left(t_{i}\right)^{2}, i=1,2, \ldots, N-1$, where $N$ is the number of sample studied. Similar to the procedure of estimating the multivariate regression by the method of least squares, we can obtain the least squares estimator of the model as

$$
\widehat{A}_{0}=\left(X^{T} X\right)^{-1} X^{T} Y
$$

where

$$
X=\left(\begin{array}{ccccc}
1 & X_{11} & X_{12} & \cdots & X_{19} \\
1 & X_{21} & X_{22} & \cdots & X_{29} \\
\vdots & \vdots & \vdots & \vdots & \vdots \\
1 & X_{(N-1) 1} & X_{(N-1) 2} & \cdots & X_{(N-1) 9}
\end{array}\right)
$$

and $Y=\left(Y_{1}, Y_{2}, \ldots, Y_{N-1}\right)^{T}$. The superscript $T$ indicates the transposition of matrix and vector.

In what follows, we consider the first regression equation and estimate the parameters $\left(q_{1}, A_{1}\right)$. The corresponding least squares estimation is subjected to

$$
\begin{aligned}
\left(\widehat{q}_{1}, \widehat{A}_{1}\right)= & \arg \min \operatorname{SSR}\left(q_{1}, A_{1}\right) \\
= & \arg \min \sum_{n=1}^{N-1}\left(\frac{x\left(t_{n+1}\right)-x\left(t_{n}\right)}{\left(t_{n+1}-t_{n}\right)^{q_{1}}} \Gamma\left(1+q_{1}\right)\right. \\
& \left.\quad-f\left(x_{t_{n}}, y_{t_{n}}, z_{t_{n}}, A_{1}\right)\right)^{2} \\
= & \arg \min \left(\Gamma\left(q_{1}+1\right)\left(t_{n+1}-t_{n}\right)^{1-q_{1}}\right)^{2} \\
& \times \sum_{n=1}^{N-1}\left(\frac{x\left(t_{n+1}\right)-x\left(t_{n}\right)}{\left(t_{n+1}-t_{n}\right)}-f\left(x_{t_{n}}, y_{t_{n}}, z_{t_{n}}, A_{1}^{\prime}\right)\right)^{2},
\end{aligned}
$$

where

$$
A_{1}^{\prime}=\frac{\left(t_{n+1}-t_{n}\right)^{q_{1}-1}}{\Gamma\left(q_{1}+1\right)} A_{1} .
$$

According to the evaluation result with $q_{1}=1$, the minimum of the second product part implies that $A_{1}^{\prime}=\widehat{A}_{0}$. Hence,

$$
\begin{aligned}
& \widehat{q}_{1}=\arg \min \left\{\Gamma\left(q_{1}+1\right)\left(t_{n+1}-t_{n}\right)^{1-q_{1}}\right\}, \\
& A_{1}^{\prime}=\widehat{A}_{0},
\end{aligned}
$$

and the minimum of $\operatorname{SSR}\left(q_{1}, A_{1}\right)$ can be obtained as

$$
\begin{aligned}
& \widehat{A}_{1}=\Gamma\left(\widehat{q}_{1}+1\right)\left(t_{n+1}-t_{n}\right)^{1-q_{1}}\left(X^{T} X\right)^{-1} X^{T} Y, \\
& \widehat{q}_{1}=\arg \min \left\{\Gamma\left(q_{1}+1\right)\left(t_{n+1}-t_{n}\right)^{1-q_{1}}\right\} .
\end{aligned}
$$

TABLE 1: Optimal fractional order $q$.

\begin{tabular}{lccccc}
\hline$\Delta t$ & 1 & 0.9 & 0.8 & 0.7 & 0.6 \\
\hline$\widehat{q}_{i}$ & 0.4616 & 0.3580 & 0.2536 & 0.1483 & 0.0416 \\
\hline
\end{tabular}

Note. When $\Delta t \leq 0.5, \widehat{q}_{i}<0$; thus the case of $\Delta t \leq 0.5$ is not to be considered.

Equations (18)-(19) are the least squares estimation of $q_{1}$ and $A_{1}$ in the first regression equation of system (9), respectively. It is easy to find that the estimator of $q_{1}$ is not related to the sample observations, and can be computed by numerically. Using the same technique, we can deal with $q_{2}$ and $q_{3}$ in system (9) and obtain the optimal estimators of $\widehat{q}_{2}$ and $\widehat{q}_{3}$. In the next section, we will consider the dynamics of our new model and the prediction results based on the macroeconomic data of Japan.

\section{Empirical Results of Discrete Fractional Financial System: Evidence from Japan}

In this section, we present the study of discrete financial system based on the macroeconomic data of Japan.

4.1. Data Description. In financial model (9), the nonlinear dynamic behaviors of interest rate, investment demand, and inflation are studied. This work chooses six-month London interbank offered rate (LIBOR) data to reflect interest rate change in Japan. The total investment percent of GDP is used to measure the investment demand. Average consumer prices percent change rate will be used to reflect the inflation. The annual data starts from year 1980 to 2011. The data about LIBOR, investment percent of GDP, and average consumer prices percent change rate are obtained from EconStats which is organized by IMF.

4.2. Empirical Results. The optimal fractional orders $q_{i}, i=$ $1,2,3$ with different step sizes $\Delta t=1,0.9,0.8,0.7,0.6$ are performed in Table 1. We do not consider the case of $\Delta t<$ 0.5 because of the fact that the fractional order decreases and approaches to zero, which reduces our model to be a linear one but not the fractional financial system.

Tables 2-4 show the results about the estimated coefficients, sum squared residuals, and $P$ values of statistical tests in the equations of empirical model under the situation of different time steps of discretization. From Table 2, we can find that the sum squared residuals are less than $5 \times 10^{-3}$ in the first equation of (9), and the $P$ values of the $F$-statistics are less than $5 \%$ in the estimation results of different time step steps, which demonstrate that the estimation results based on the empirical data are reasonable statistically. Moreover, the time step sizes of discretization do not influence the $P$ values of $t$-test for coefficients and $P$ value of $F$-statistics for the empirical model. Finally, the structure of terms at which corresponding coefficients estimated are significant at $5 \%$ level in the empirical equation about interest rate includes terms $y, z, y z, z x, x^{2}$, constant term, and is independent of the variation of the time step sizes of discretization. 
TABLE 2: The first equation in (9) under the several time steps of discretization.

\begin{tabular}{|c|c|c|c|c|c|}
\hline & & & $\Delta t$ & & \\
\hline & 0.6 & 0.7 & 0.8 & 0.9 & 1 \\
\hline$c$ & $\begin{array}{c}-0.08301^{* *} \\
(0.0227)\end{array}$ & $\begin{array}{c}-0.0818^{* *} \\
(0.0227)\end{array}$ & $\begin{array}{c}-0.0797^{* *} \\
(0.0227)\end{array}$ & $\begin{array}{c}-0.0769^{* *} \\
(0.0227)\end{array}$ & $\begin{array}{c}-0.0736^{* *} \\
(0.0227)\end{array}$ \\
\hline$x$ & & & & & \\
\hline$y$ & $\begin{array}{c}0.3933^{* *} \\
(0.0131)\end{array}$ & $\begin{array}{c}0.3876^{* *} \\
(0.0131)\end{array}$ & $\begin{array}{c}0.3774^{* *} \\
(0.0131)\end{array}$ & $\begin{array}{c}0.3641^{* *} \\
(0.0131)\end{array}$ & $\begin{array}{c}0.3488 \\
(0.0131)\end{array}$ \\
\hline$z$ & $\begin{array}{l}4.0327^{* *} \\
(0.0481)\end{array}$ & $\begin{array}{c}3.9749^{* *} \\
(0.0481)\end{array}$ & $\begin{array}{c}3.8703^{* *} \\
(0.0481)\end{array}$ & $\begin{array}{l}3.7338^{* *} \\
(0.0481)\end{array}$ & $\begin{array}{l}3.5763^{* *} \\
(0.0481)\end{array}$ \\
\hline$x y$ & & & & & \\
\hline$y z$ & $\begin{array}{c}-21.8019^{* *} \\
(0.0128)\end{array}$ & $\begin{array}{c}-21.4897^{* *} \\
(0.0128)\end{array}$ & $\begin{array}{c}-20.9243^{* *} \\
(0.0128)\end{array}$ & $\begin{array}{c}-20.1863^{* *} \\
(0.0128)\end{array}$ & $\begin{array}{c}-19.3349^{* *} \\
(0.0128)\end{array}$ \\
\hline$z x$ & $\begin{array}{c}29.9767^{* * *} \\
(0.0010)\end{array}$ & $\begin{array}{c}29.5474^{* * *} \\
(0.0010)\end{array}$ & $\begin{array}{c}28.7700^{* * *} \\
(0.0010)\end{array}$ & $\begin{array}{c}27.7553^{* * *} \\
(0.0010)\end{array}$ & $\begin{array}{c}26.5846^{* * *} \\
(0.0010)\end{array}$ \\
\hline$x^{2}$ & $\begin{array}{c}-6.1145^{* * *} \\
(0.0002)\end{array}$ & $\begin{array}{c}-6.0269^{* * *} \\
(0.0002)\end{array}$ & $\begin{array}{c}-5.8684^{* * *} \\
(0.0002)\end{array}$ & $\begin{array}{c}-5.6614^{* * *} \\
(0.0002)\end{array}$ & $\begin{array}{c}-5.4226^{* *} \\
(0.0002)\end{array}$ \\
\hline$y^{2}$ & & & & & \\
\hline$z^{2}$ & & & & & \\
\hline SSR & $4.5 e-3$ & $4.3 e-3$ & $4.2 e-3$ & $3.9 e-3$ & $3.6 e-3$ \\
\hline $\operatorname{Prob}(F)$ & 0.0068 & 0.0068 & 0.0068 & 0.0068 & 0.0068 \\
\hline
\end{tabular}

Note. $* * *, * *$, and $*$ denote statistical significance at the $1 \%, 5 \%$, and $10 \%$ levels. SSR is the sum squared residuals. Prob is the $P$ value of $F$-statistic.

TABLE 3: The second equation in (9) under the several time steps of discretization.

\begin{tabular}{|c|c|c|c|c|c|}
\hline & & & $\Delta t$ & & \\
\hline & 0.6 & 0.7 & 0.8 & 0.9 & 1 \\
\hline . & $0.2419^{* *}$ & $0.2384^{* *}$ & $0.2321^{* *}$ & $0.2239^{* *}$ & $0.2145^{* *}$ \\
\hline 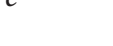 & $(0.0448)$ & $(0.0448)$ & $(0.0448)$ & $(0.0448)$ & $(0.0448)$ \\
\hline$x$ & $0.6527^{* *}$ & $0.6434^{* *}$ & $0.6264^{* *}$ & $0.6043^{* *}$ & $0.5789^{* *}$ \\
\hline$x$ & $(0.0123)$ & $(0.0123)$ & $(0.0123)$ & $(0.0123)$ & $(0.0123)$ \\
\hline$y$ & $-2.1378^{* *}$ & $-2.1071^{* *}$ & $-2.0517^{* *}$ & $-1.9793^{* *}$ & $-1.8958^{* *}$ \\
\hline$y$ & $(0.0255)$ & $(0.0255)$ & $(0.0255)$ & $(0.0255)$ & $(0.0255)$ \\
\hline$z$ & & & & & \\
\hline$x y$ & & & & & \\
\hline$\gamma z$ & $-5.4639^{* * *}$ & $-5.3856^{* * *}$ & $-5.2439^{* * *}$ & $-5.0590^{* * *}$ & $-4.8456^{* * *}$ \\
\hline$y z$ & $(0.0035)$ & $(0.0035)$ & $(0.0035)$ & $(0.0035)$ & $(0.0035)$ \\
\hline$\sigma x$ & $10.8938^{* *}$ & $10.7378^{* *}$ & $10.4553^{* *}$ & $10.0865^{* *}$ & $9.6611^{* *}$ \\
\hline $2 x$ & $(0.0282)$ & $(0.0282)$ & $(0.0282)$ & $(0.0282)$ & $(0.0282)$ \\
\hline$x^{2}$ & $-4.2457^{* *}$ & $-4.1849^{* *}$ & $-4.0748^{* *}$ & $-3.9311^{* *}$ & $-3.7653^{* *}$ \\
\hline & $(0.0197)$ & $(0.0197)$ & $(0.0197)$ & $(0.0197)$ & $(0.0197)$ \\
\hline$v^{2}$ & $4.2928^{* *}$ & $4.2313^{* *}$ & $4.1200^{* *}$ & $3.9747^{* *}$ & $3.8070^{* *}$ \\
\hline$y$ & $(0.0179)$ & $(0.0179)$ & $(0.0179)$ & $(0.0179)$ & $(0.0179)$ \\
\hline$z^{2}$ & & & & & \\
\hline SSR & $2.0 e-3$ & $2.0 e-3$ & $1.9 e-3$ & $1.7 e-3$ & $1.6 e-3$ \\
\hline $\operatorname{Prob}(F)$ & $(0.0179)$ & $(0.0179)$ & $(0.0179)$ & 0.0180 & 0.0180 \\
\hline
\end{tabular}

Note. $* * *, *$, and $*$ denote statistical significance at the $1 \%, 5 \%$, and $10 \%$ levels. SSR is the sum squared residuals. Prob is the $P$ value of $F$-statistic. 
TABLE 4: The third equation in (9) under the several time steps of discretization.

\begin{tabular}{|c|c|c|c|c|c|}
\hline & & & $\Delta t$ & & \\
\hline & 0.6 & 0.7 & 0.8 & 0.9 & 1 \\
\hline . & $0.2528^{* * *}$ & $0.2528^{* * *}$ & $0.2426^{* * *}$ & $0.2341^{* * *}$ & $0.2242^{* *}$ \\
\hline$c$ & $(0.0041)$ & $(0.0041)$ & $(0.0041)$ & $(0.0041)$ & $(0.0041)$ \\
\hline$x$ & $0.1387^{* *}$ & $0.1386^{* *}$ & $0.1331^{* *}$ & $0.1284^{* *}$ & $0.1230^{* *}$ \\
\hline$\lambda$ & $(0.0350)$ & $(0.0350)$ & $(0.0350)$ & $(0.0350)$ & $(0.0350)$ \\
\hline$y$ & $\begin{array}{c}-2.1169^{* * *} \\
(0.0025)\end{array}$ & $\begin{array}{c}-2.1166^{* * *} \\
(0.0025)\end{array}$ & $\begin{array}{c}-2.0317^{* * *} \\
(0.0025)\end{array}$ & $\begin{array}{c}-1.9601^{* * *} \\
(0.0025)\end{array}$ & $\begin{array}{c}-1.8774^{* * *} \\
(0.0025)\end{array}$ \\
\hline$z$ & & & & & \\
\hline$x y$ & & & & & \\
\hline$y z$ & $\begin{array}{c}-2.8366^{* * *} \\
(0.0000)\end{array}$ & $\begin{array}{c}-2.8361^{* * *} \\
(0.0000)\end{array}$ & $\begin{array}{c}-2.7224^{* * *} \\
(0.0000)\end{array}$ & $\begin{array}{c}-2.6264^{* * *} \\
(0.0000)\end{array}$ & $\begin{array}{c}-2.5156^{* * *} \\
(0.0000)\end{array}$ \\
\hline$z x$ & & & & & \\
\hline$x^{2}$ & & & & & \\
\hline$y^{2}$ & $\begin{array}{c}4.2750^{* * *} \\
(0.0017)\end{array}$ & $\begin{array}{c}4.2743^{* * *} \\
(0.0017)\end{array}$ & $\begin{array}{c}4.1029^{* * *} \\
(0.0017)\end{array}$ & $\begin{array}{c}3.9582^{* * *} \\
(0.0017)\end{array}$ & $\begin{array}{l}3.7912^{* *} \\
(0.0017)\end{array}$ \\
\hline$z^{2}$ & & & & & \\
\hline SSR & $1.3 e-3$ & $1.3 e-3$ & $1.2 e-3$ & $1.1 e-3$ & $1.0 e-3$ \\
\hline $\operatorname{Prob}(\mathrm{F})$ & 0.0000 & 0.0000 & 0.0000 & 0.0000 & 0.0000 \\
\hline
\end{tabular}

Note. $* * *, * *$, and $*$ : denote statistical significance at the $1 \%, 5 \%$, and $10 \%$ levels. SSR is the sum squared residuals. Prob is the $P$ value of $F$-statistic.

The result is consistent with the fact that the estimation of $q_{i}$ is not related to the sample observations in (19).

In Table 3, we observe that the sum squared residuals are less than $2 \times 10^{-3}$ for the empirical equation of the second equation of (9), and the $P$ values of the $F$-statistics are 0.0068 which is less than $5 \%$, which also demonstrate that the estimation results about the second equation of (9) are reasonable statistically. The result that the time step sizes of discretization do not influence the $P$ values can also be found in Table 3. Moreover, the structure of terms at which corresponding coefficients estimated are significant at 5\% level in the empirical equation about interest rate includes terms $x, y, y z, z x, x^{2}, y^{2}$, constant term. Similar to the first empirical equation, and it is not influenced by the variation of the time step sizes of discretization.

In Table 4, we observe that the sum squared residuals are less than $1.5 \times 10^{-3}$ for the empirical equation of the third equation of (9), which suggests that the accuracy of fitness about the third empirical equation is satisfying. The $P$ values of the $F$-statistics are 0.0000 which is less than $5 \%$, which demonstrate that the estimation of the third equation is reasonable in the statistical sense. Moreover, the structure of terms at which corresponding coefficients estimated are significant at $5 \%$ level in the empirical equation about interest rate includes terms $x, y, y z, y^{2}$, constant term. Both of the structure of terms and $P$ values of the coefficients are not changing with the variation of the time step sizes of discretization.

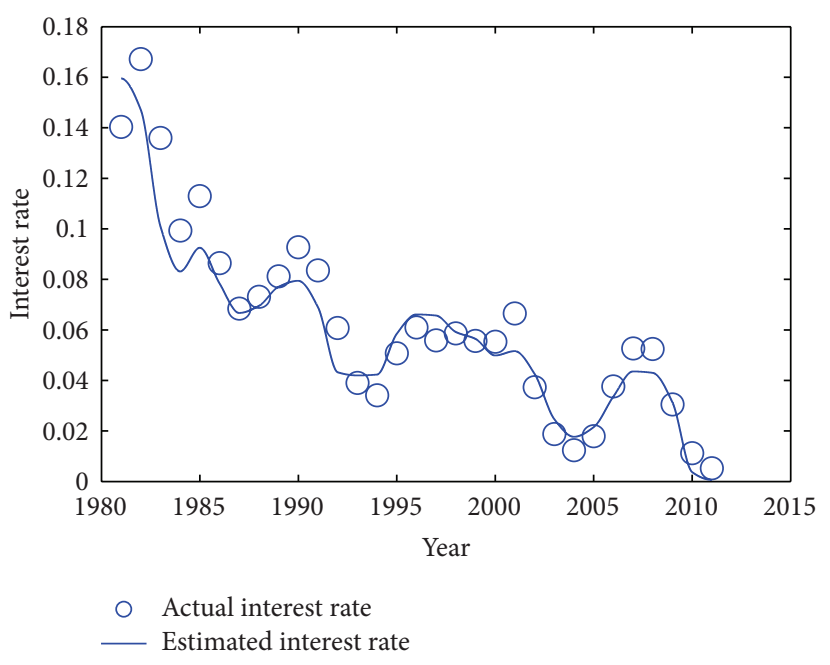

Figure 1: The actual interest rate versus estimated interest rate.

Figures 1-3 show the data estimated by the empirical model in case of $\Delta t=1$ and the actual data of the interest rate, investment, and inflation, respectively. In Figure 1, we can find that the blue line passes through the vast majority of the circle except those in the initial years. According to the circles and blue line which depict the estimated data and actual data of the interest rate respectively, we find that the empirical equation of the interest rate in the model can 


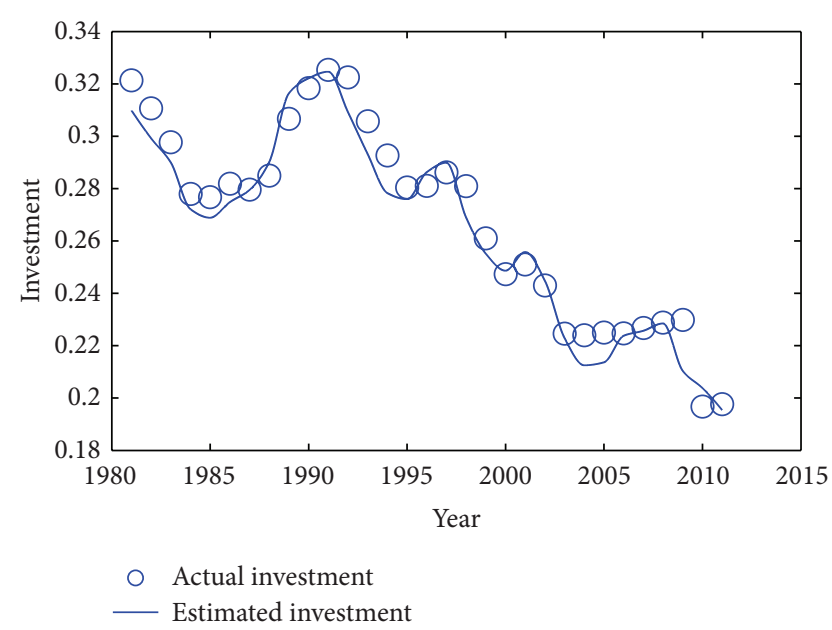

FIGURE 2: The actual investment versus estimated investment.

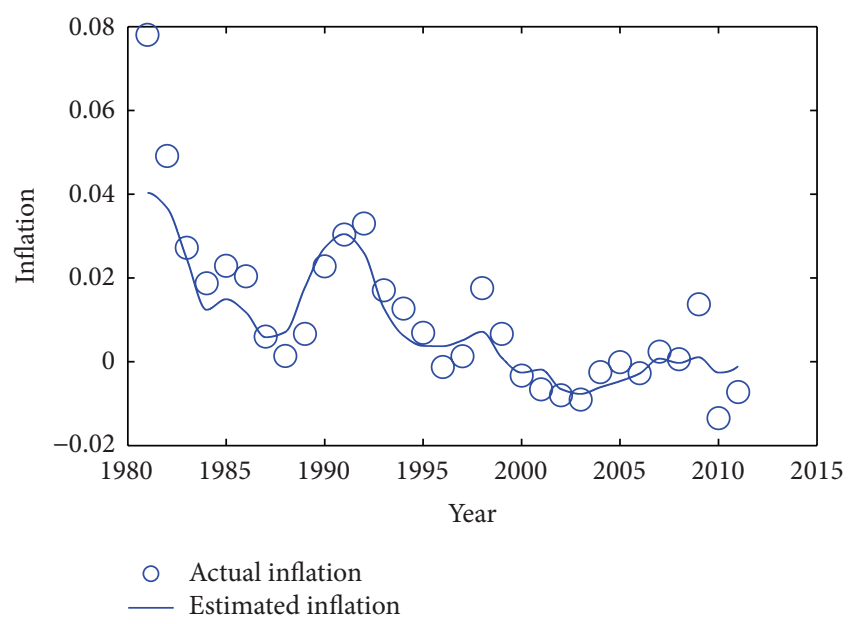

FIGURE 3: The actual inflation versus estimated inflation.

describe the actual data effectively. In Figure 2, the blue line almost passes through all of the circle notations. The property of the figure declares that the actual investment data can be fitted by the empirical equation accurately. In Figure 3, there are only very few circle notations that deviate from the blue line in the beginning and ending parts of the period. Figure 3 suggests that the empirical equation about inflation fits the actual data ideally. Above all, Figures 1-3 suggest that the empirical model estimated by the methodology proposed in the paper depicts the actual data reasonably.

In order to analyze the effectiveness of the consequence about the prediction of the empirical model, the multistep predictions of interest rate, investment, and inflation in the case that $\Delta t=1$ are shown in Figures 4-6, respectively. From Figure 4, we can find that the predictions are very close to the actual interest rate from the black line which corresponds to the four-step predictions, and then the bias of predictions from the actual data increases with the steps of prediction increasing, such as purple, yellow, and green line. It suggests that the prediction about interest rate is meaningful in the first four years, and the accuracy of predictions becomes more

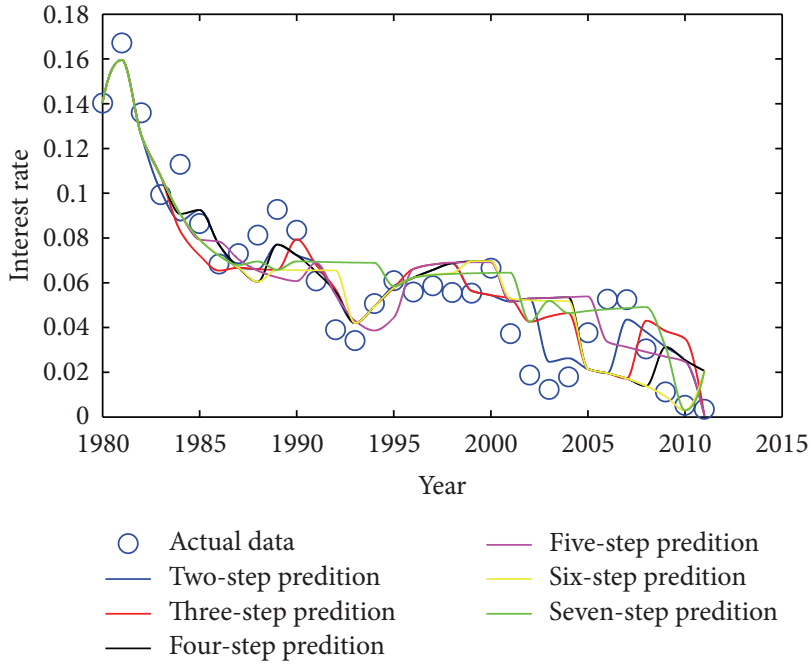

FIGURE 4: The predictions of interest rate based on empirical model in the case of $\Delta t=1$.

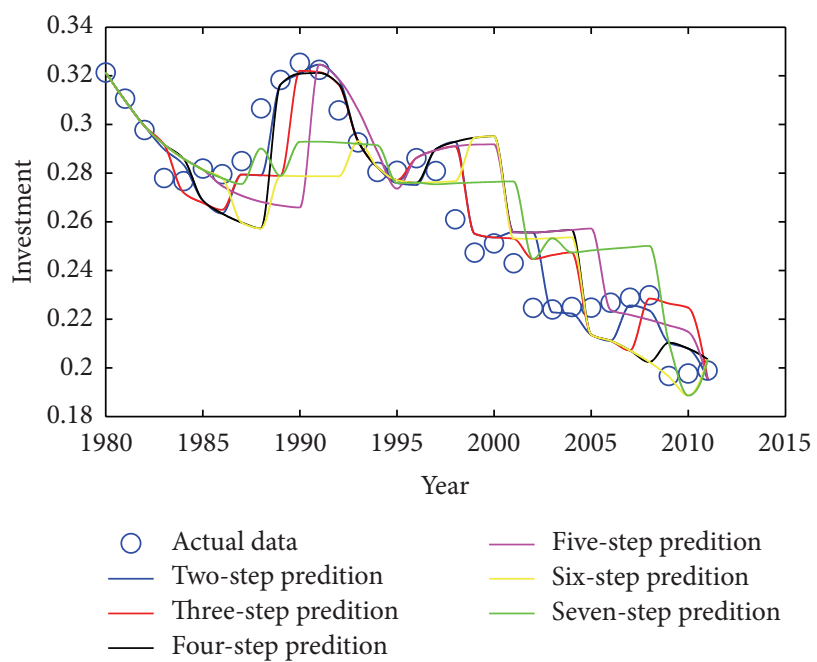

FIGURE 5: The predictions of investment based empirical model in the case of $\Delta t=1$.

frustrating when the time steps of the prediction are more than four time steps. In Figure 5, the bias of the prediction about investment increases as the time steps increase, but the prediction with a few steps still approaches the actual data, such as the black line which is corresponding to the four-step prediction. In Figure 6, we also can find that the accuracy of the prediction of the inflation becomes unsatisfying in the situations that the time step of prediction is not less than four, but the predictions with steps being less than four are close to the actual inflation. The above results suggest that the four-step prediction of the empirical model estimated is meaningful for interest rate, investment, and inflation.

\section{Conclusions}

In this paper we propose a new nonlinear dynamic financial econometric model by using the Jumarie's fractional-order 


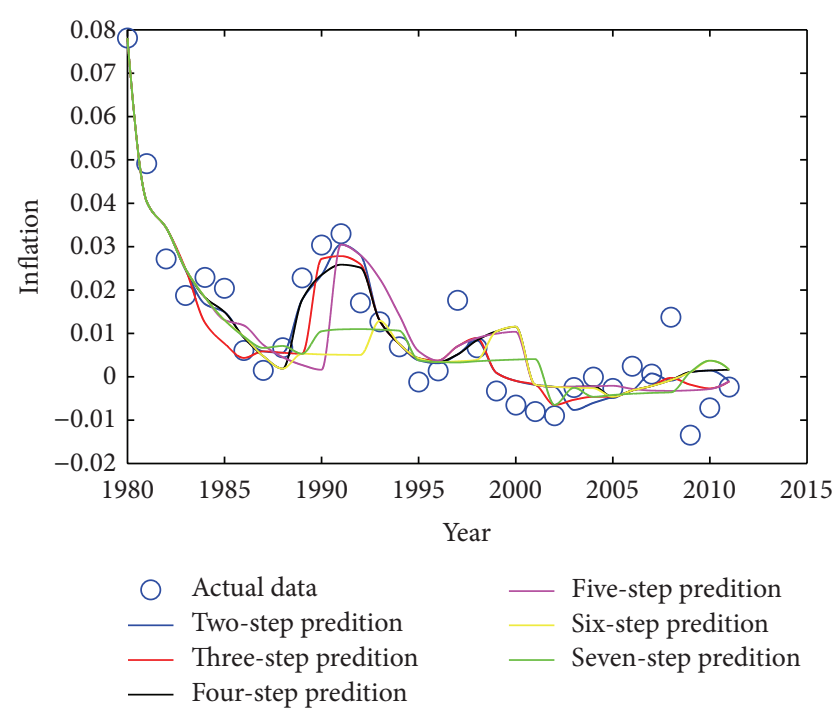

FIGURE 6: The predictions of inflation based on empirical model in the case of $\Delta t=1$.

derivative. The corresponding discrete financial model is generated by removing the limit operation in the Jumarie's derivative. The model overcomes the problems which cannot be depicted by some simplified nonlinear financial model in literatures, and it provides a feasible technique for describing the actual macroeconomic data of one particular place by nonlinear model. Our model is proved to be reasonable via empirical analysis.

Based on the macroeconomic data of Japan, we evaluate the parameters of the financial model. The suitable fractional order for Japan's data is obtained. In the empirical study, we observe that the fractional order has an apparent influence on the dynamics behavior of financial system. With the optimal fractional order, our new fractional financial model can be used to predict the dynamic behavior of financial system of Japan in the coming years reasonably.

\section{Acknowledgment}

This work is supported by the National Natural Science Foundation of China (Grant no. 70873136).

\section{References}

[1] R. Goodwin, Chaotic Economic Dynamics, Oxford University Press, New York, NY, USA, 1990.

[2] R. Shone, An Introduction to Economic Dynamics, Cambridge University Press, Cambridge, Mass, USA, 1997.

[3] R. Shone, Economic Dynamics, Cambridge University Press, Cambridge, Mass, USA, 2nd edition, 2002.

[4] J. Stachurski, Economic Dynamics, MIT Press, Cambridge, Mass, USA, 2009, Theory and Computation.

[5] A. C. Chian, "Nonlinear dynamics and chaos in macroeconomics," International Journal of Theoretical and Applied Finance, vol. 3, no. 3, pp. 601-601, 2000.

[6] A. C.-L. Chian, F. A. Borotto, E. L. Rempel, and C. Rogers, "Attractor merging crisis in chaotic business cycles," Chaos, Solitons and Fractals, vol. 24, no. 3, pp. 869-875, 2005.
[7] A. C.-L. Chian, E. L. Rempel, and C. Rogers, "Complex economic dynamics: chaotic saddle, crisis and intermittency," Chaos, Solitons and Fractals, vol. 29, no. 5, pp. 1194-1218, 2006.

[8] L. De Cesare and M. Sportelli, "A dynamic IS-LM model with delayed taxation revenues," Chaos, Solitons and Fractals, vol. 25, no. 1, pp. 233-244, 2005.

[9] L. Fanti and P. Manfredi, "Chaotic business cycles and fiscal policy: an IS-LM model with distributed tax collection lags," Chaos, Solitons and Fractals, vol. 32, no. 2, pp. 736-744, 2007.

[10] J. H. Ma and Y. S. Chen, "Study for the bifurcation topological structure and the global complicated character of a kind of nonlinear finance system. I," Applied Mathematics and Mechanics, vol. 22, no. 11, pp. 1119-1128, 2001.

[11] J. H. Ma and Y. S. Chen, "Study for the bifurcation topological structure and the global complicated character of a kind of nonlinear finance system. II," Applied Mathematics and Mechanics, vol. 22, no. 12, pp. 1236-1242, 2001.

[12] H.-W. Lorenz and H. E. Nusse, "Chaotic attractors, chaotic saddles, and fractal basin boundaries: goodwin's nonlinear accelerator model reconsidered," Chaos, Solitons and Fractals, vol. 13, no. 5, pp. 957-965, 2002.

[13] I. Podlubny, Fractional Differential Equations, vol. 198, Academic Press, San Diego, Calif, USA, 1999.

[14] A. A. Kilbas, H. M. Srivastava, and J. J. Trujillo, Theory and Applications of Fractional Differential Equations, vol. 204, Elsevier, Amsterdam, The Netherlands, 2006.

[15] K. Diethelm, The Analysis of Fractional Differential Equations, vol. 2004, Springer, Berlin, Germany, 2010.

[16] Y. Xu and Z. He, "The short memory principle for solving Abel differential equation of fractional order," Computers \& Mathematics with Applications, vol. 62, no. 12, pp. 4796-4805, 2011.

[17] Y. F. Xu and Z. M. He, "Existence and uniqueness results for Cauchy problem of variable-order fractional differential equations," Journal of Applied Mathematics and Computing, vol. 43, no. 1-2, pp. 295-306, 2013.

[18] A. Kirman and G. Teyssiere, "Microeconomic models for long memory in the volatility of financial time series," Studies in Nonlinear Dynamics \& Econometrics, vol. 5, no. 4, pp. 281-302, 2002.

[19] K. Yamasaki, L. Muchnik, S. Havlin, A. Bunde, and H. E. Stanley, "Scaling and memory in volatility return intervals in financial markets," Proceedings of the National Academy of Sciences of the United States of America, vol. 102, no. 26, pp. 9424-9428, 2005.

[20] V. Anh and A. Inoue, "Financial markets with memory. I. Dynamic models," Stochastic Analysis and Applications, vol. 23, no. 2, pp. 275-300, 2005.

[21] F. Garzarelli, M. Cristelli, A. Zaccaria, and L. Pietronero, "Memory effects in stock price dynamics: evidences of technical trading," preprint 2011, http://arxiv.org/abs/1110.5197.

[22] W.-C. Chen, "Nonlinear dynamics and chaos in a fractionalorder financial system," Chaos, Solitons and Fractals, vol. 36, no. 5, pp. 1305-1314, 2008.

[23] S. Dadras and H. R. Momeni, "Control of a fractional-order economical system via sliding mode," Physica A, vol. 389, no. 12, pp. 2434-2442, 2010.

[24] M. Salah Abd-Elouahab, N.-E. Hamri, and J. Wang, "Chaos control of a fractional-order financial system," Mathematical Problems in Engineering, vol. 2010, Article ID 270646, 18 pages, 2010. 
[25] Z. Wang, X. Huang, and G. Shi, "Analysis of nonlinear dynamics and chaos in a fractional order financial system with time delay," Computers \& Mathematics with Applications, vol. 62, no. 3, pp. 1531-1539, 2011.

[26] S. Ma, Y. Xu, and W. Yue, "Numerical solutions of a variableorder fractional financial system," Journal of Applied Mathematics, vol. 2012, Article ID 417942, 14 pages, 2012.

[27] G. Jumarie, "Modified Riemann-Liouville derivative and fractional Taylor series of nondifferentiable functions further results," Computers \& Mathematics with Applications, vol. 51, no. 9-10, pp. 1367-1376, 2006. 


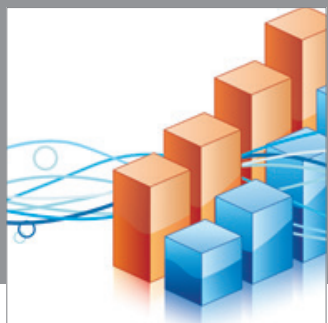

Advances in

Operations Research

mansans

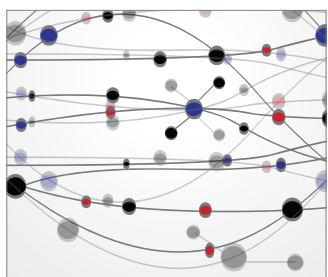

The Scientific World Journal
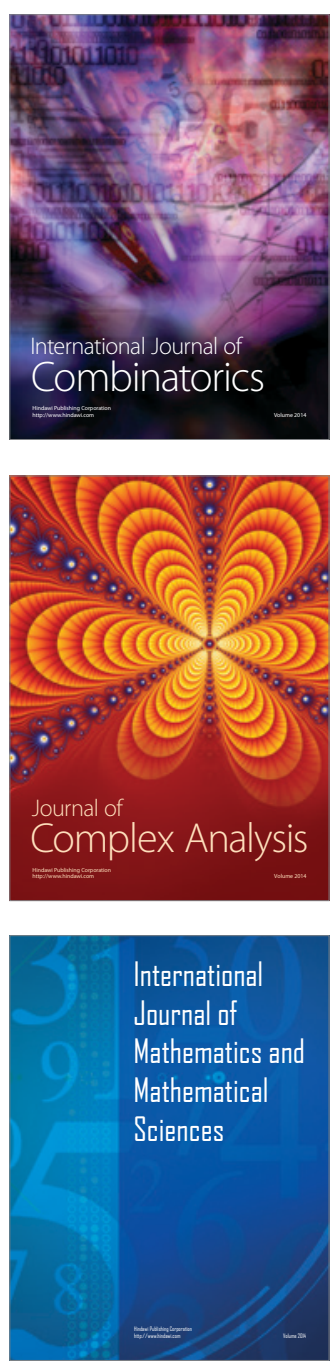
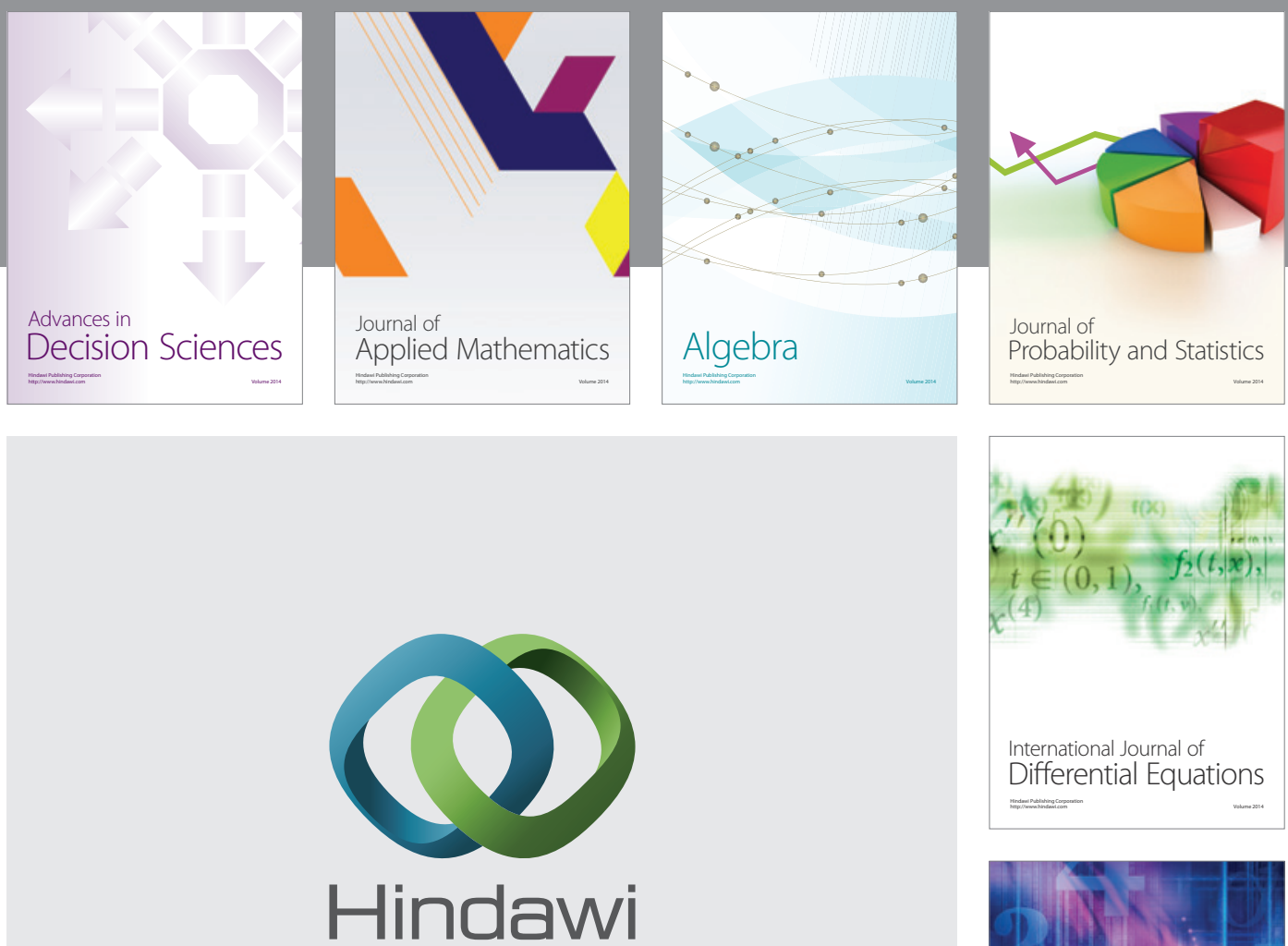

Submit your manuscripts at http://www.hindawi.com
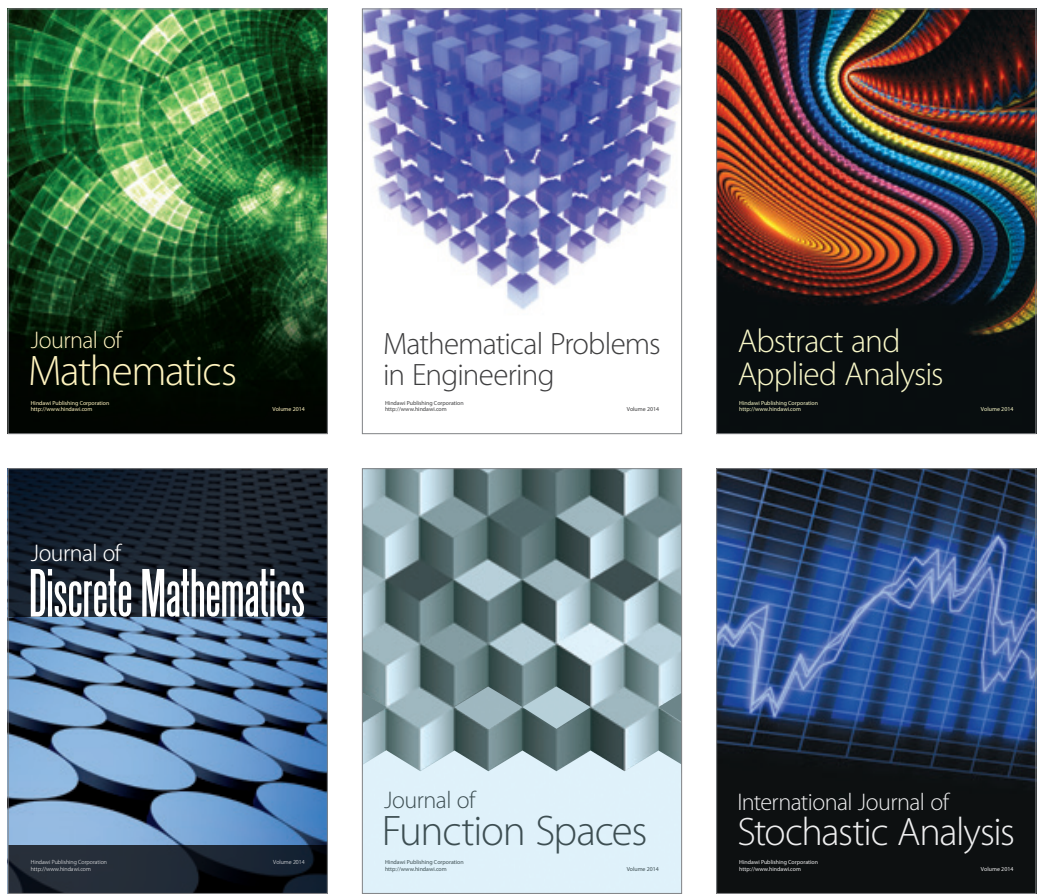

Journal of

Function Spaces

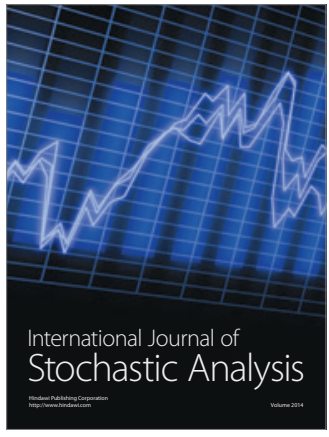

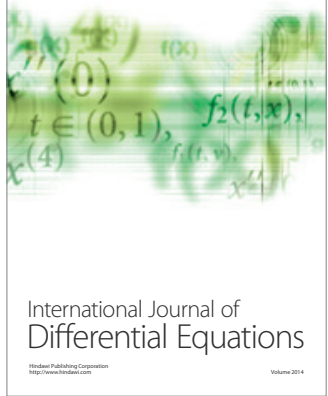
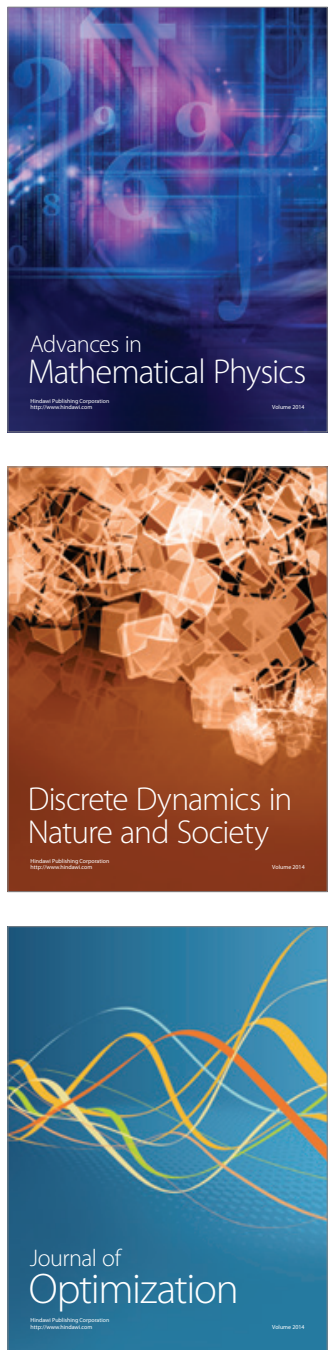